\title{
Robust Fuzzy PSS Design using ABC
}

\author{
O. Abedinia* \\ Islamic Azad University, \\ Young Researcher Club, Ardabil \\ Branch, Ardabil, Iran \\ E-mail: oveis.abedinia@gmail.com
}

\author{
B. Wyns \\ Department of Electrical Energy, \\ Systems and Automation \\ Ghent, Belgium \\ E-mail: bart.wyns@ugent.be
}

\author{
A. Ghasemi \\ Technical Engineering Department, \\ University of Mohaghegh Ardabili, \\ Ardabil, Iran \\ E-mail: alighasemi1365@gmail.com
}

\begin{abstract}
This paper presents an Artificial Bee Colony (ABC) algorithm to tune optimal rule-base of a Fuzzy Power System Stabilizer (FPSS) which leads to damp low frequency oscillation following disturbances in power systems. Thus, extraction of an appropriate set of rules or selection of an optimal set of rules from the set of possible rules is an important and essential step toward the design of any successful fuzzy logic controller. Consequently, in this paper, an $\mathrm{ABC}$ based rule generation method is proposed for automated fuzzy PSS design to improve power system stability and reduce the design effort. The effectiveness of the proposed method is demonstrated on a 3machine 9-bus standard power system in comparison with the Genetic Algorithm based tuned FPSS under different loading condition through ITAE performance indices.
\end{abstract}

Keywords: PSS, Fuzzy PSS Design, ABC, Multi-machine Power Systems, Rule-base.

\section{INTRODUCTION}

Electro-mechanical oscillations on the transmission grid are becoming a critical problem for the power systems. There are two distinct kinds of dynamic oscillations which have been known to present problems on power systems. One type occurs when a generating unit (group of units) at a station is (are) swinging against the rest of the system. Such oscillations are called local mode oscillations. The characteristic frequency of a typical local mode is generally in the $1-2 \mathrm{~Hz}$ range, depending mainly on the impedance of the transmission system [1]. The second type of oscillations, known as inter-area modes, are more complex because they usually involve a combination of many machines on one part of a system swinging against machines on another part of the system. The characteristic frequency of inter-area modes of oscillations is generally in the range of 0.1 to $0.6 \mathrm{~Hz}$. A Power System Stabilizer (PSS) is an electronic feedback control that is a part of the excitation system control for generating units. PSS acts to modulate the generator field voltage to damp power system oscillations [1].

A number of techniques have been introduced for deigning PSSs in recent decades. Conventionally lead-lag control is one of the traditional methods. The main problem encountered in the Conventional PSS (CPSS) design is the power system constantly experiences changes in operating conditions due to variation in generation and load patterns, as well as changes in transmission networks. Hence, the achieved results of this method exhibit poor dynamic performance [2].

Genetic algorithms (GA) are stochastic search techniques based on the mechanism of natural selection and survival of the fittest which is independent on the complexity of problems where no prior knowledge is available [3]. Literature reveals many contributions using GAs for simultaneous stabilization of multi-machine power systems over a wide range of scenarios via PSS with fixed parameters. Although a GA is very efficient in finding global or near global optimal solution to the problem, it requires a very long computation time depending on the size of the system under study [4].

Fuzzy systems deal with a set of linguistic rules, which are put down by experienced operators. It is a model-free approach, which is generally considered suitable for controlling imprecisely defined systems. In this method, the controller is synthesized from a collection of fuzzy If-Then rules which describe the behavior of the unknown plant. Fuzzy PSSs (FPSSs) has been applied in a number of publications [5]. In [6] the parameters of the FPSS are kept fixed after the design is completed. The performance of the FPSS depends on the operating conditions of the power system, although it is less sensitive than CPSSs.

In order to overcome these problems, an ABC algorithm based PID type controller is proposed as a solution to the PSS problem. Here, the ABC optimization algorithm is used for the optimal tuning of the fuzzy rule-base to improve the optimization synthesis and damping low frequency oscillations following disturbances in power systems. The ABC algorithm is a typical swarm-based approach to optimization, in which the search algorithm is inspired by the intelligent foraging behavior of a honey bee swarm process [7] and has emerged as a useful tool for engineering optimization. It incorporates a flexible and well-balanced mechanism to adapt to the global and local exploration and exploitation abilities within a short computation time. Hence, this method is efficient in handling large and complex search spaces [8].

The 3-9 buses standard power system, under various system configurations and loading conditions is employed to illustrate the effectiveness of the proposed method in comparison with the GAPSS [4] and robust GA based tuned fuzzy controller [5] through nonlinear time simulation and some performance indices. Results show that the proposed method is effective and alternative to conventional fixed gain stabilizer design as it retains the simplicity of the CPSS and still guarantees a robust acceptable performance over a wide range of operating and system conditions.

\section{A. Case Study}

\section{PROBLEM STATEMENT}

The 3-machine 9-bus power system shown in "Fig.1", is employed for this study. The system is tested in various operating points; i.e. nominal, lightly and heavily conditions. The system and load conditions data are given in [5].

\section{B. Fuzzy PID Controller Design}

Recently the research for control methods based on Fuzzy Logic Controllers (FLC) as PSS has greatly improved the dynamic characteristics of power system [9]. Membership functions' shape and fuzzy rules should be adjusted to obtain the best control performance in FLC. Conventionally the

* Corresponding author. This work is sponsored by Young Researcher Club, Ardabil Branch. 
adjustment is done by experts or by trial and error methods. Therefore it is difficult to determine the suitable membership functions and rule-base without the knowledge of the system. These problems make the design process more difficult [9-10]. On the other hand, FLC robustness and reliability make fuzzy controllers useful for solving a wide range of control problems in power systems. In general, the application of fuzzy logic to PID control design for the PSS design can be classified in two major categories according to the way of their construction [5]:

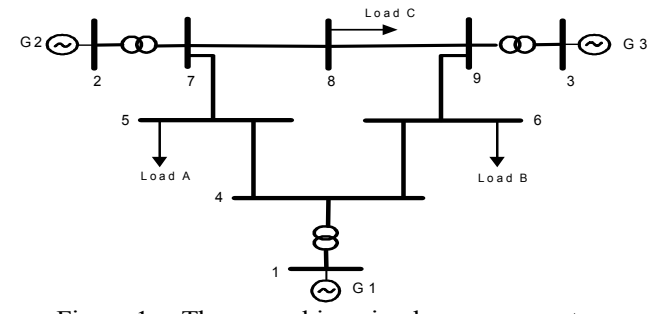

Figure 1. Three-machine nine-bus power system.

1. A typical PSS is constructed as a set of heuristic control rules, and the control signal is directly deduced from the knowledge base.

2. The gains of the conventional PID controller are tuned online in terms of the knowledge base and fuzzy inference, and then, the conventional PID controller generates the control signal.

The fuzzy inference process comprises the following five steps:

1. Fuzzification of the input variables.

2. Application of the fuzzy operator (AND or OR) in the antecedents.

3. Implication from the antecedent to the consequent.

4. Aggregation of the consequents across the rules.

5. Defuzzification.

The block diagram of the classical fuzzy type controller to PSS design for each generator is presented in "Fig. 2".

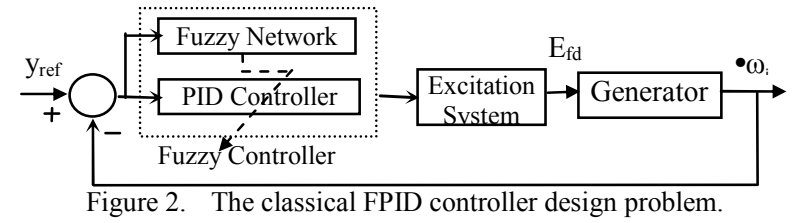

The classical FPID controller includes two stages. The first one is the fuzzy network and the second level is the PID controller. The controller block is formed by fuzzification of $\left(\Delta \omega_{i}\right)$, the interface mechanism and defuzzification. Therefore $\mathrm{u}_{\mathrm{i}}$ is a control signal which applies to the excitation system in each generator. By taking $\Delta \omega_{\mathrm{i}}$ as the system output, the control vector for the conventional PID controller is given by:

$$
u_{i}=K_{P i i} \Delta \omega_{i}(t)+K_{I i} \int_{0}^{t} \Delta \omega_{i}(t) d t+K_{d i} \Delta \dot{\omega}_{i}(t)
$$

The $\mathrm{K}_{\mathrm{Ii}}, \mathrm{K}_{\mathrm{di}}$ and $\mathrm{K}_{\mathrm{pi}}$ parameters are achieved by a set of fuzzy rules which are defined as:

If $\Delta \omega_{i}$ is $A_{i}$ and $\Delta\left(\Delta \omega_{i}\right)$ is $B_{i}$ then $K_{d i}$ is $C_{i}$ and $K_{p i}$ is $D_{i}$ and $K_{\text {Ii }}$ is $E_{i}, i=1,2, \cdots, n$.

Where, $A_{i}, B_{i}, C_{i}, D_{i}$ and $E_{i}$ are fuzzy sets on the corresponding supporting sets.

According to the FLC design, and depending on the performance of the FPID controller it should be considered that an intelligent design method for FPID control, which generates optimal rule tables without human experts, is desirable. In this study an $\mathrm{ABC}$ technique is utilized to find optimal rule sets of the FPID based PSS.

\section{Artificial Bee Colony Algorithm}

The ABC algorithm is proposed by Karaboga [7] in 2005, and the performance of $\mathrm{ABC}$ is analyzed in 2007 [11]. The foraging bees are classified into three categories; employed bees, onlookers and scout bees. All bees that are currently exploiting a food source are known as employed. The employed bees exploit the food source and they carry the information about food source back to the hive and share this information with onlooker bees. Onlookers bees are waiting in the hive for the information to be shared by the employed bees about their discovered food sources and scouts bees will always be searching for new food sources near the hive. Employed bees share information about food sources by dancing in the designated dance area inside the hive. The nature of dance is proportional to the nectar content of food source just exploited by the dancing bee. Onlooker bees watch the dance and choose a food source according to the probability proportional to the quality of that food source [7]. Therefore, good food sources attract more onlooker bees compared to bad ones. Whenever a food source is exploited fully, all the employed bees associated with it abandon the food source, and become scout. Scout bees can be visualized as performing the job of exploration, whereas employed and onlooker bees can be visualized as performing the job of exploitation [8].

In the $\mathrm{ABC}$ algorithm, the number of employed bees is equal to the number of food sources which is also equal to the number of onlooker bees. There is only one employed bee for each food source whose first position is randomly generated. Each employed bee, in each iteration of the algorithm determines a new neighboring food source of its currently associated food source by "Eq. (2)", and computes the nectar amount of this new food source:

$$
v_{i j}=z_{i j}+\theta_{i j}\left(z_{i j}-z_{k j}\right)
$$

Where, $\mathrm{k} \in\{1,2, \ldots, \mathrm{BN}\}$ and $\mathrm{j} \in\{1,2, \ldots, \mathrm{D}\}$ are randomly chosen indexes. Although $\mathrm{k}$ is determined randomly, it has to be different from i. $\theta_{\mathrm{ij}}$ is a random number between $[-1,1]$. It controls the production of a neighbor food source position around $\mathrm{z}_{\mathrm{ij}}$. The modification represents the comparison of the neighbor food positions visually by the bee. "Eq. (2)" shows that as the difference between the parameters of the $z_{i j}$ and $z_{k j}$ decreases, the perturbation on the position $\mathrm{z}_{\mathrm{ij}}$ decreases, too. If the nectar amount of this new food source is higher than that of its currently associated food source, then this employed bee moves to this new food source, otherwise it continues with the old one. After all employed bees complete the search process; they share the information about their food sources with onlooker bees [11]. An onlooker bee evaluates the nectar information taken from all employed bees and chooses a food source with a probability related to its nectar amount by "Eq. (3)". This method, known as roulette wheel selection method, provides better candidates to have a greater chance of being selected:

$$
p_{i}=f i t_{i} /\left(\sum_{n=1}^{S N} f i t_{i}\right)
$$


Where fit $t_{i}$ is the fitness value of the solution $i$ which is proportional to the nectar amount of the food source in the position $\mathrm{i}$ and $\mathrm{SN}$ is the number of food sources which is equal to the number of employed bees. The pseudo code for $\mathrm{ABC}$ algorithm looks as follows:

- Initialize

- Repeat.

- Move the employed bees onto their food source and evaluate the fitness

- Move the onlookers onto the food source and evaluate their fitness

- Move the scouts for searching new food source

- Memorize the best food source found so far

- Until (termination criteria satisfied)

\section{ABC TO DESIGN FUZZY CONTROLLER}

In this paper, an ABC- Fuzzy based on PSS (ABCFPSS) is proposed, which combines the advantage of the $\mathrm{ABC}$ and fuzzy control techniques to achieve good robust performance. It should be mentioned that obtaining the optimal decisionmaking logic for the proposed ABC fuzzy control strategy is very important to achieve the desired level of robust performance [9], because it is a computationally expensive combinatorial optimization problem. Usually, the rule-base sets are determined by experience and control knowledge of a human expert. However, experts may not always be available and even when available it is a trial-and-error process that takes much time and cost [9]. In order to overcome this drawback and to reduce the fuzzy system design effort and cost, a modified ABC technique is being used to construct the optimal rule-base sets of the proposed fuzzy type PID controller. "Fig. 3", shows the structure of the proposed ABCFPSS to improve power system stability. The results of the fuzzy rule-base sets are listed in tables 1-3. Accordingly the rule result for $\mathrm{K}_{\mathrm{Pi}}$ will be same as the $\mathrm{K}_{\mathrm{Di}}$. Also the parameters of $\mathrm{ABC}$ algorithm are: number of rules (40), Max iteration (100) and optimal fitness value (0.032).

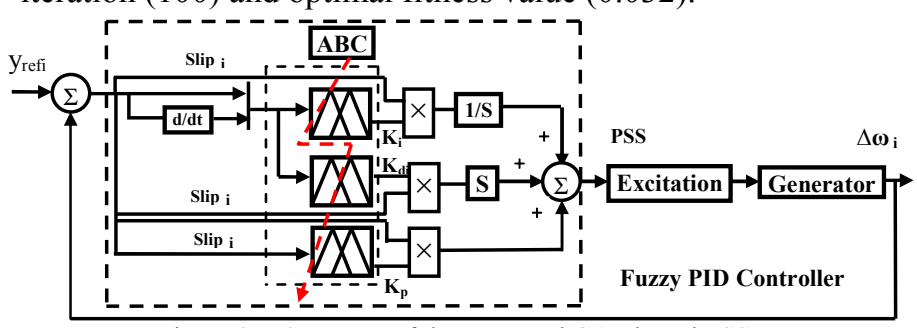

Figure 3. Structure of the proposed GAF based PSS.

In the proposed rule-base optimization problem, the membership function sets for the $\mathrm{K}_{\mathrm{Pi}}, \mathrm{K}_{\mathrm{Ii}}$ and $\mathrm{K}_{\mathrm{di}}$ are defined as triangular partitions with five segments from 0 to 1 as shown in Fig. 4. Zero $(\mathrm{ZO})$ is the center membership function. The remaining parts of the partition are Negative Big (NB), Negative Small (NS), Positive Small (PS) and Positive Big (PB). The membership function sets for $\Delta \omega i, \Delta(\Delta \omega i)$ are the same as Membership Function (MF) sets as shown in "Fig. 4". The PSS is connected to machines G2 and G3 in the test system. Here, the modified ABC evolution procedure is applied to produce rule tables of the proposed controller to guarantee relative stability and concurrently secure the time domain specifications.

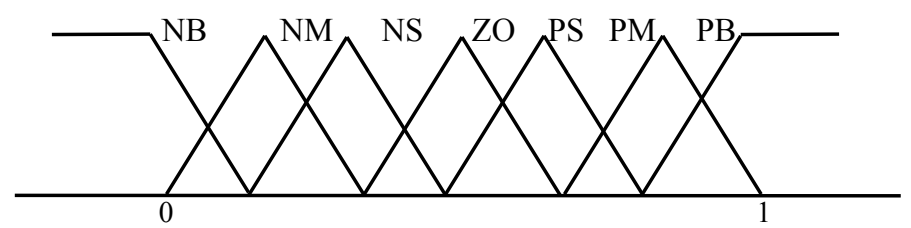

Figure 4. The MF sets for $\mathrm{K}_{\mathrm{Pi}}, \mathrm{K}_{\mathrm{Ii}}$ and $\mathrm{K}_{\mathrm{di}}$.

Evaluation of the Integral of the Time multiplied Absolute value of the Error (ITAE) based function is an alternative of the conventional maximization of fitness function, which defined in [5]. "Fig. 5", shows the plot of obtained fitness function value.

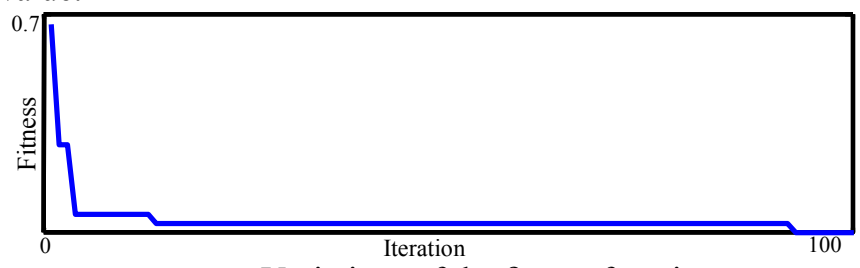

Figure 5. Variations of the fitness functions. TABLE I. OPTIMAL RULE-BASE FOR $\mathrm{K}_{\mathrm{II}}$

\begin{tabular}{|l|llll|}
\hline & NB & NS & PS & PB \\
\hline NB & PM & NS & PM & ZO \\
NS & NM & NB & ZO & ZO \\
Z & NM & PB & ZO & PB \\
PS & NS & PM & NS & NM \\
PB & PM & NB & NS & NS \\
\hline
\end{tabular}

\begin{tabular}{|l|llll|}
\multicolumn{2}{c}{} & \multicolumn{2}{c|}{ TABLE II. } & \multicolumn{3}{l|}{ OPTIMAL RULE-BASE FOR K $\mathrm{KI}$} \\
\hline & NB & NS & PS & PB \\
\hline NB & PM & NS & NM & PB \\
NS & PM & ZO & NB & ZO \\
Z & PM & NM & PM & PM \\
PS & NS & PM & PM & NB \\
PB & NM & PB & NB & NS \\
\hline
\end{tabular}

\begin{tabular}{|l|llll|}
\multicolumn{3}{c|}{ TABLE III. } & \multicolumn{3}{l|}{ OPTIMAL RULE-BASE FOR $\mathrm{K}_{\mathrm{PI}}$} \\
\hline & NB & NS & PS & PB \\
\hline NB & PM & NS & NM & PB \\
NS & PM & ZO & NB & ZO \\
Z & PM & NM & PM & PM \\
PS & NS & PM & PM & NB \\
PB & NM & PB & NB & NS \\
\hline
\end{tabular}

A. Nonlinear time-domain simulation

To demonstrate the effectiveness and robustness of the proposed ABCFPSS, simulation studies are carried out under fault disturbances and fault clearing sequences for two scenarios. The performance of the proposed controller is compared to those of the PSSs tuned using the GA method [4] and GAFPSS [5] for different operating conditions. The numerical results of ITAE are presented in table 4.

\begin{tabular}{|c|ccc|ccc|}
\multicolumn{4}{c}{ TABLE IV. } & \multicolumn{3}{c|}{ VALUE OF ITAE IN DIFFERENT TECHNIQUES } \\
\cline { 2 - 7 } Method & \multicolumn{3}{|c|}{ Scenario 1 } & \multicolumn{3}{c|}{ Scenario 2 } \\
\cline { 2 - 7 } & \multicolumn{2}{|c|}{ Nominal Light } & Heavy & Nominal & Light & Heavy \\
\hline ABC-FPSS & 0.62 & 0.61 & 0.67 & 0.6 & 0.56 & 0.65 \\
GA-FPSS & 0.76 & 0.73 & 0.84 & 0.73 & 0.70 & 0.79 \\
TSPSS & 37.1 & 36.7 & 38.5 & 36.03 & 36 & 37.4 \\
\hline
\end{tabular}

- Scenario 1

In this scenario, a 0.1 p.u. step increase in the mechanical torque of three machines was applied at $\mathrm{t}=1.0 \mathrm{~s}$. The speed deviations of the generators under heavy loading conditions are shown in "Fig. 6". It can be seen that the ABCFPSSs achieves good robust performance and provides superior damping in comparison with the other methods. 

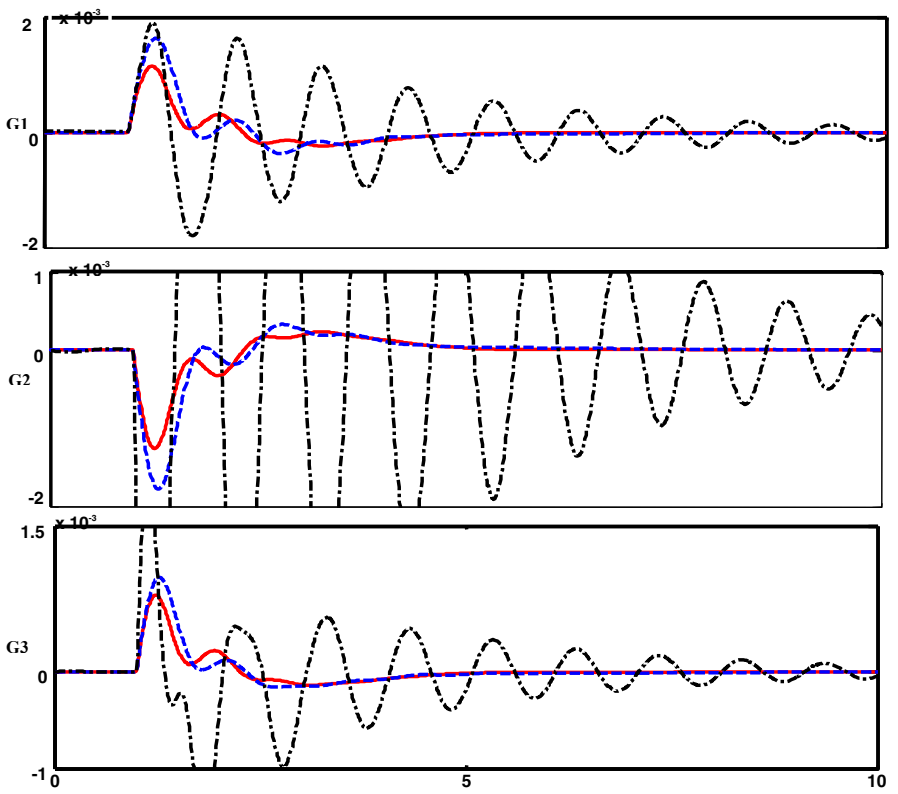

Figure 6. System response under scenario 1 with heavy loading condition: Solid (ABCFPSS) Dashed (GAFPSS) Doted (GAPSS).

- Scenario 2

In this scenario performance of the proposed controller under transient conditions is verified by applying a 6-cycle three-phase fault at $\mathrm{t}=1 \mathrm{sec}$, on bus 7 at the end of the line 5-7. The fault is cleared by permanent tripping the faulted line. "Fig.7" shows the speed deviations of machines under the nominal loading conditions. It can be seen that the overshoot, undershoot, settling time and speed deviations of all machines are greatly reduced by applying the proposed ABCFPSSs.
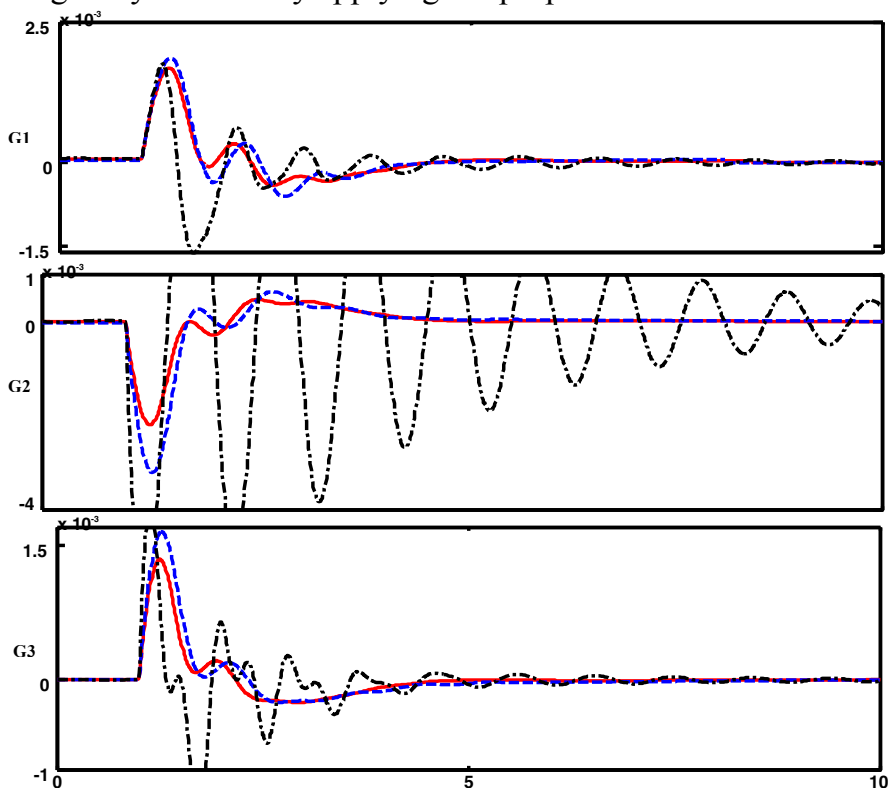

Figure 7. System response under scenario 2 with nominal loading condition: Solid (ABCFPSS) Dashed (GAFPSS) Doted (GAPSS).

\section{CONCLUSION}

In this paper, a new robust ABC based fuzzy PSS is proposed to enhance relative stability and secure operation of a multi machine power system. The motivation of using the proposed ABCFPSS is to reduce the fuzzy system design effort and determine the optimal controller scheme such that the relative stability is guaranteed and the time domain specifications are concurrently secured. The ABC algorithm is a search algorithm that is inspired by the intelligent foraging behavior of a honey bee swarm process and has emerged as a useful tool for engineering optimization. It incorporates a flexible and well-balanced mechanism to adapt to the global and local exploration and exploitation abilities within a short computation time. Hence, this method is efficient in handling large and complex search spaces. The proposed control strategy in this contribution combines the advantage of the fuzzy control system and $\mathrm{ABC}$ techniques for achieving the desired level of robust performance under different operating conditions and load disturbances through ITAE performance indices. An important feature of the proposed method is that it does not require an accurate model of the system. All PSSs are designed simultaneously, by taking into consideration the interaction among them. Furthermore, they have a simple and decentralized nature since only local measurements are employed as the stabilizer inputs. This makes the ABCFPSS easy to implement and ideally useful for the real world power systems. The effectiveness of the proposed method is tested on 3-machine 9-buses power system for a wide range of load demands and disturbances under different operating conditions. The nonlinear time simulation results confirm that the proposed ABCFPSS can work effectively over a wide range of loading conditions and is superior to the GAFPSS and GAPSS.

\section{REFERENCES}

[1] M. J. Basler, R. C. Schaefer, "Understanding power system stability", IEEE Trans. Industry Applications, vol. 44, pp. 463-474, 2008.

[2] Kundur, "Power system stability and control", New York: McGrawHill; 1994.

[3] O. Abedinia, M. Salay Naderi, A. Jalili, B. Khamenehpour, "Optimal tuning of multi-machine power system stabilizer parameters using genetic-algorithm", IEEE Transaction on Power Systems, unpublished.

[4] JM. Call, "Genetic algorithms for modeling and optimization", Journal of Computational and Applied on Mathematics, vol. 184, pp. 205-222, 2005.

[5] HA. Shayanfar, H. Shayeghi, O. Abedinia, A. Jalili, "Design rule-base of fuzzy controller in multi-machine power system stabilizer using genetic algorithm", In: Proceedings of the international conference on artificial intelligence, Las Vegas, Nevada, USA, pp. 43-49, July 2010.

[6] El. Zonkoly, AA. Khalil, NM. Ahmied, "Optimal tuning of lead-lag and fuzzy logic power system stabilizers using particle swarm optimization", Expert System with Applications, vol. 10, pp. 1-10, 2008.

[7] D. Karaboga, "An idea based on honey bee swarm for numerical optimization", Technical Report-TR06, Erciyes University, Engineering Faculty, Computer Engineering Department, 2005.

[8] SL. Sabat, SK. Udgata, A. Abraham, "Artificial bee colony algorithm for small signal model parameter extraction of MESFET, Engineering Applications of Artificial Intelligence, pp. 1-6, 2010.

[9] H. Shayeghi, HA. Shayanfar, O. Abedinia, Mohammad S. Naderi, "Robust design of fuzzy power system stabilizer via GA", IEEE Transaction on Power Systems, Arizona, USA, unpublished.

[10] H. Shayeghi, H.A. Shayanfar, A. Jalili, A. Ghasemi, "LFC design using HBMO technique in interconnected power system", International Journal on "Technical and Physical Problems of Engineering" (IJTPE), vol. 1, no. 4, pp. 41-48, 2010.

[11] D. Karaboga, B. Basturk, "A powerful and efficient algorithm for numerical function optimization: Artificial Bee Colony (ABC) algorithm", Journal of Global Optimization, Springer Netherlands, vol.39, no.3, pp.459-471, 2007. 